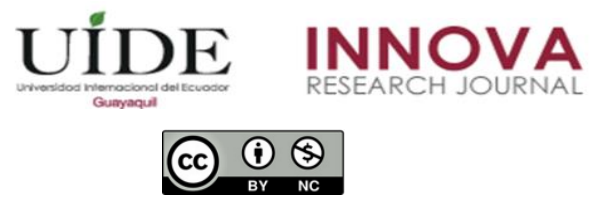

INNOVA Research Journal, ISSN 2477-9024

(Enero-Abril 2020). Vol. 5, No.1 pp. 14-30

DOI: https://doi.org/10.33890/innova.v5.n1.2020.1036

URL: http://revistas.uide.edu.ec/index.php/innova/index

Correo: innova@uide.edu.ec

\title{
La psicología positiva aplicada en el proceso de evaluación docente de la Pontificia Universidad Católica del Ecuador - Ambato
}

\section{Positive psychology applied in the process of teacher evaluation of the Pontificia Universidad Católica del Ecuador - Ambato}

Teresa Milena Freire Aillón

Galo Mauricio López Sevilla

Diego Armando Jiménez Bósquez

Pontificia Universidad Católica del Ecuador, Ecuador

Autor por correspondencia: tfreire@pucesa.edu.ec; glopez@ pucesa.edu.ec;

djimenez@pucesa.edu.ec

Fecha de recepción: 02 de junio del 2019 - Fecha de aceptación: 22 de octubre del 2019

\section{Resumen}

El artículo presenta una propuesta para fortalecer el proceso de evaluación docente en la Pontificia Universidad Católica del Ecuador Ambato (PUCESA), desde el enfoque de la psicología positiva con las fortalezas de carácter, para procurar el bienestar en los docentes. La normativa de Educación Superior ecuatoriana, reglamenta la evaluación al personal docente, así, la institución ha dispuesto procesos y modelos de evaluación, cuya aplicación ha registrado en los últimos años, dificultades como la resistencia al proceso de evaluación por parte de sus actores. Debe desarrollarse entonces, en un marco adecuado y motivador para que los docentes alcancen estándares, con el compromiso y sentido de pertenencia que genera el aplicar mecanismos de control dentro de un marco positivo y humano, orientado a la mejora continua profesional y personal. La investigación tiene un alcance exploratorio y descriptivo, con un enfoque mixto, basado en una investigación no experimental, se utilizó el análisis de información en lo que respecta a reglamentos, perfiles docentes y modelos, y se aplicó una encuesta para recopilar información. La propuesta incorpora las fortalezas de carácter al perfil de competencias del docente de la PUCESA, así como actividades orientadas a desarrollar fortalezas de carácter ideales para los evaluados y evaluadores.

Palabras Claves: evaluación docente; psicología positiva; fortalezas de carácter, calidad educativa

\begin{abstract}
The article presents a proposal to strengthen the process of teacher evaluation at the Pontifical Catholic University of Ecuador Ambato (PUCESA), from the perspective of positive psychology with strengths of character, to ensure the welfare of teachers. The Ecuadorian Higher Education regulations, regulate the evaluation of teaching staff, so the institution has established evaluation processes and models, whose application has registered in recent years, difficulties such as resistance to the evaluation process by its actors. It must be developed then, in an appropriate and
\end{abstract}


motivating framework for teachers to reach standards, with the commitment and sense of belonging generated by applying control mechanisms within a positive and human framework, aimed at continuous professional and personal improvement. The research has an exploratory and descriptive scope, with a mixed approach, based on a non-experimental investigation, the analysis of information regarding regulations, teaching profiles and models was used, and a survey was applied to gather information. The proposal incorporates the strengths of character to the profile of competencies of the teacher of PUCESA, as well as activities aimed at developing strengths of an ideal nature for those evaluated and evaluators.

Key words: teacher evaluation; positive psychology; strengths of character, educational quality

\section{Introducción}

De manera general, la evaluación se entiende como un proceso orientado a observar, apreciar y emitir un juicio respecto a alguna determinada situación o cosa, esto con el fin de lograr a corto, mediano o largo plazo una mejora en aquello que es objeto de la evaluación.

Se considera un proceso integral puesto que involucra aspectos éticos y técnicos, en donde, si bien es importante la medición, la comparación y el control, busca además provocar un diálogo orientado a estimular y mejorar una situación, identificando de manera crítica las causas que llevaron a que dicha situación se refleje como está (Blázquez, 2007).

En educación superior, los docentes desarrollan sus actividades alrededor de varios ámbitos: docencia, investigación, vinculación, gestión académica, entre otros, es allí en donde la evaluación del desempeño docente toma una enorme importancia, para garantizar que el aporte que realizan al cumplimiento de los estándares e indicadores institucionales responde a los parámetros de calidad requeridos.

El marco normativo que rige la Educación Superior en Ecuador establece la necesidad de evaluar al personal docente. En razón de estos requerimientos y de su naturaleza, la PUCESA, ha dispuesto distintos procesos y modelos de evaluación de su personal académico. Esta aplicación de modelos técnicos de evaluación ha registrado, en los últimos años, una serie de dificultades que se han evidenciado en una creciente resistencia al proceso de evaluación, tanto en el personal evaluado como en quienes evalúan.

La resistencia a este proceso de evaluación tiene varias explicaciones, entre ellas, se consideran, las que se ha observado son las más recurrentes y comunes: primero, los evaluados ven en este proceso un mecanismo de seguimiento cuya finalidad es perseguir a los profesores y justificar posibles despidos; segundo, no hay una cultura de evaluación, pues a los evaluados y a los evaluadores les cuesta asimilar objetivamente el proceso y verlo como una oportunidad para mejorar y corregir lo que debe ser corregido; y tercero, los estudiantes no se sienten parte del proceso de evaluación docente, razón por la cual su participación es bastante indiferente.

La resistencia y sus implicaciones al proceso de evaluación docente lo convierten en un problema mayor en la vida de la Universidad, esto porque la evaluación, al ser rechazada y ejecutada con una mala actitud, no cumple con el objetivo de identificar oportunidades de mejora 
en la calidad de las actividades docentes y en contribuir a la generación de un ambiente laboral amigable y de confianza mutua.

Es entonces fundamental que los procesos de evaluación docente se desarrollen dentro de un marco adecuado, propicio, motivador para que los docentes sientan la necesidad de alcanzar estándares e indicadores establecidos, no por cumplir un requerimiento institucional, sino llevados por el compromiso y sentido de pertenencia que genera el aplicar mecanismos de control dentro de una marco positivo y humano, orientado a la mejora continua tanto en la parte profesional como personal.

La presente propuesta se enmarca en mejorar la percepción de dicho proceso, de manera que reconociendo la naturaleza participativa de distintos actores en diversos procesos evaluativos se busca darle un enfoque sincero y de justicia con el objeto de disminuir la subjetividad de la evaluación, aportando elementos que puedan ser aplicables a partir de la experiencia de la educación positiva.

\section{Revisión de la literatura}

\section{Modelo de evaluación docente de la PUCE}

De acuerdo a lo que establece el "Modelo de Evaluación Institucional de Universidades y Escuelas Politécnicas 2018” (CACES, 2018), la Pontificia Universidad Católica del Ecuador (PUCE), en el mes de Enero de 2018, puso en vigencia el Modelo de Evaluación Integral del Personal Académico (MEIPA) en todas sus sedes, incluida la sede Ambato, el mismo que se fundamenta en el aprendizaje basado en competencias y pretende "de manera sistemática evaluar y medir la efectividad y gestión de los docentes, a través del cumplimiento de sus responsabilidades, manteniendo el comportamiento adecuado y esperado" (PUCE, 2018, p. 3).

Con la aplicación de este modelo de evaluación, la PUCESA se proyecta a realizar planes de desarrollo orientados a la mejora de la calidad de los docentes con el fin de tener personal calificado.

Para comprender de mejor manera la metodología que el MEIPA utiliza dentro del proceso de evaluación docente, se presenta a continuación la figura 1: 


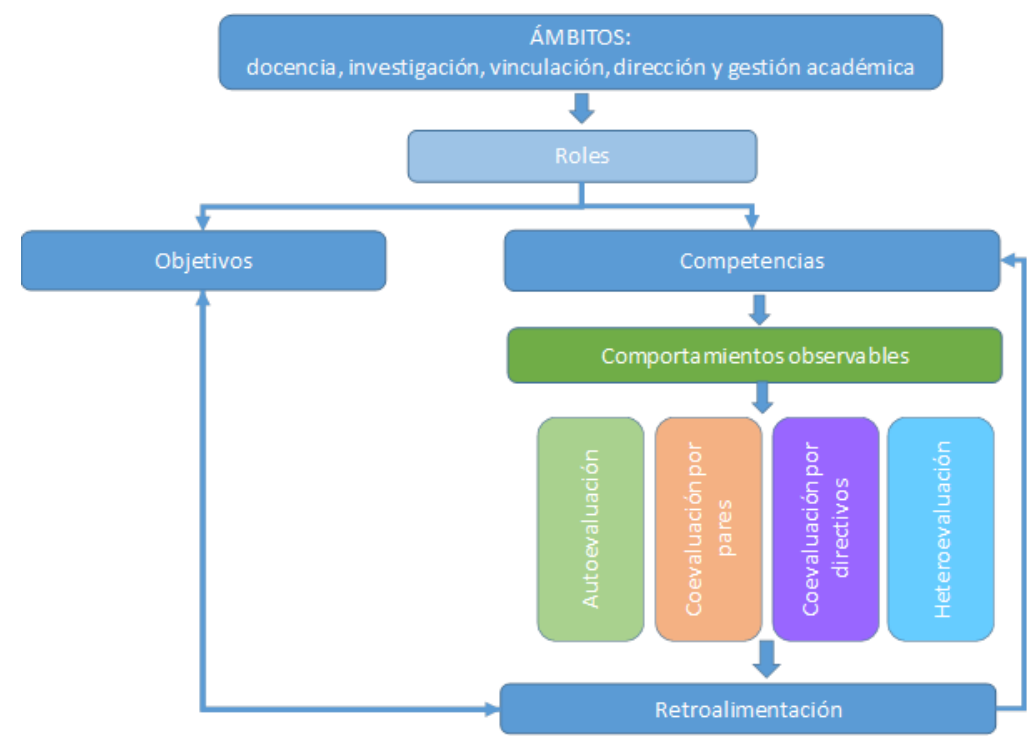

Figura \#1 detalla el esquema general del Modelo de Evaluación Integral del Personal Académico de la PUCE (PUCE, 2018).

El modelo de evaluación, responde al Reglamento General de Personal Académico y Escalafón de la PUCE (2017), respecto a los ámbitos de evaluación, en donde se establece que el docente debe ser evaluado en sus actividades de docencia, investigación, vinculación, dirección y gestión académica. Dentro de estos ámbitos, se han especificado distintos roles, los cuales permiten definir las actividades de las que son responsables los docentes además de las competencias que deben cumplir según el perfil requerido, especificado en el Diccionario de Competencias del personal académico de la PUCE (2018)

Para cada rol se han especificado varios instrumentos de evaluación, los cuales están estructurados con una serie de comportamientos observables dentro de los distintos momentos de evaluación (autoevaluación, co evaluación, y hetero evaluación), estos comportamientos observables están vinculados a una serie de competencias que se han definido en el Diccionario de Competencias del personal académico del PUCE (2018), que reflejan el perfil ideal del Docente de la PUCE y son evaluados en función de escalas de Likert de frecuencia o acuerdo en un rango de 0 a 4.

El modelo de evaluación incorpora un protocolo a ser aplicado antes, durante y después de la evaluación docente, que comprende actividades orientadas a garantizar la objetividad del proceso dentro del marco del Modelo Educativo de la PUCE, el mismo que define a la evaluación como un mecanismo orientado a alcanzar la calidad y mejora continua de la educación, pero en íntima relación a la transformación personal, institucional y social (PUCE, 2017).

Los resultados del proceso de evaluación finalmente se emiten en función de ponderaciones y fórmulas también incorporados en el modelo, que buscan equilibrar las horas de dedicación que cada docente tenga en sus distributivos de trabajo frente a cada uno de los roles que desempeña de manera proporcional. 
El informe final muestra los resultados de dos maneras: un resultado global, basado en una fórmula de evaluación integral existente en el modelo, en un rango de $0 \%$ a $100 \%$; otro resultado basado en las competencias que de manera cualitativa pretende reflejar el nivel de desarrollo de las competencias en un rango de 1 a 100.

El proceso culmina con la entrega de los resultados a los docentes a manera de retroalimentación que es un punto determinante para lograr un efecto positivo en los evaluados.

\section{Perfil de competencias del docente de la PUCE}

Según lo establece el MEIPA (2018), las competencias "son el conjunto de capacidades, habilidades, destrezas, conocimientos, comportamientos, atributos, actitudes y valores que una persona posee y le permiten desempeñarse de manera exitosa" (p.4), en concordancia a ello, el Diccionario de Competencias del Personal Académico de la PUCE (2018), determina el perfil de competencias que un docente de la PUCE debe tener para desarrollar su labor educativa de una manera óptima y acorde al modelo educativo de la institución.

Se han establecido 19 competencias divididas en dos grandes grupos: competencias genéricas y competencias pedagógicas, en cada una de ellas se ha establecido una explicación que permita comprender las capacidades, habilidades y actitudes a las que hace referencia; de la misma forma, se han definido escalas de valoración de frecuencia con su respectiva rúbrica para lograr de una manera objetiva, valorar su desarrollo cuando sea el caso.

En la tabla 1 se muestran las competencias con su descriptor, los mismos que han sido tomados de manera textual del Diccionario de Competencias de la PUCE:

Tabla. 1

Perfil de competencias del Docente de la PUCE

\begin{tabular}{|c|c|c|}
\hline No. & Competencia & Descriptor \\
\hline 1 & $\begin{array}{l}\text { Competencia: Autocontrol } \\
-\mathrm{AU}\end{array}$ & Dominio que tiene la persona sobre sí mismo. \\
\hline 2 & $\begin{array}{l}\text { Capacidad de aprender y } \\
\text { actualizarse } \\
\text { permanentemente - CAP }\end{array}$ & $\begin{array}{l}\text { Capacidad de buscar y asimilar nueva información que permita el desarrollo } \\
\text { de los conocimientos, competencias y aspectos actitudinales y que puedan ser } \\
\text { aplicados de forma eficaz en las actividades que desarrolla el docente }\end{array}$ \\
\hline 3 & $\begin{array}{l}\text { Capacidad de planificación } \\
\text { y de organización - CPO }\end{array}$ & $\begin{array}{l}\text { Es la capacidad de definir adecuadamente la unidad de enseñanza, tanto en } \\
\text { sus contenidos como en los métodos de enseñanza propuestos, las } \\
\text { actividades, los aprendizajes esperados y las evaluaciones programadas, } \\
\text { incluyendo el seguimiento y verificación de la información }\end{array}$ \\
\hline 4 & $\begin{array}{l}\text { Compromiso con la calidad } \\
\text { - COM }\end{array}$ & $\begin{array}{l}\text { Es el interés por mejorar de manera continua los procesos que se llevan a } \\
\text { cabo en los servicios que presta la institución, con el fin de satisfacer las } \\
\text { exigencias de estudiantes, docentes, trabajadores y usuarios. }\end{array}$ \\
\hline 5 & $\begin{array}{l}\text { Comunicación efectiva } \\
\text { (oral y escrita) - CE }\end{array}$ & $\begin{array}{l}\text { Habilidad de expresar e interpretar conceptos, pensamientos, hechos y } \\
\text { opiniones de forma oral y escrita de la manera más adecuada. }\end{array}$ \\
\hline 6 & $\begin{array}{l}\text { Ética y valores } \\
\text { (institucionales) - EV }\end{array}$ & $\begin{array}{l}\text { Obrar en todo momento de acuerdo con los valores éticos y morales, las } \\
\text { buenas costumbres y prácticas profesionales, considerando las políticas } \\
\text { institucionales. }\end{array}$ \\
\hline
\end{tabular}




\begin{tabular}{|c|c|c|}
\hline No. & Competencia & Descriptor \\
\hline \multicolumn{3}{|c|}{ Genéricas } \\
\hline 7 & $\begin{array}{l}\text { Aplicación de TIC's u otros } \\
\text { elementos para fomentar } \\
\text { interactividad en clase - } \\
\text { ATIC }\end{array}$ & $\begin{array}{l}\text { Se refiere a la incorporación en la enseñanza universitaria, según el caso, del } \\
\text { uso de las TIC's, o recursos como talleres, laboratorios o elementos que } \\
\text { fomenten la interactividad en clase y faciliten el aprendizaje del alumno, } \\
\text { desarrollando así el pensamiento crítico, la creatividad y la innovación. }\end{array}$ \\
\hline 8 & $\begin{array}{l}\text { Manejo de relaciones } \\
\text { interpersonales - MRI }\end{array}$ & $\begin{array}{l}\text { Es la habilidad de escuchar activamente, mostrar empatía, comprensión, ser } \\
\text { sensible y comprender los puntos de vista de los demás, aunque no se } \\
\text { expresen verbalmente o se expresen parcialmente. Disponer de habilidades } \\
\text { sociales y comunicativas que le permitan al docente expresar opiniones, } \\
\text { intereses y pensamientos, de manera asertiva que genere confianza en sus } \\
\text { compañeros de trabajo y alumnos }\end{array}$ \\
\hline 9 & $\begin{array}{l}\text { Identificación y resolución } \\
\text { de problemas - OS }\end{array}$ & $\begin{array}{l}\text { Se refiere a la capacidad de identificar un problema u obstáculo desde su } \\
\text { origen hasta el resultado que se desea obtener; además de la capacidad para } \\
\text { proponer soluciones con diferentes procedimientos y métodos que se } \\
\text { utilizarán para la resolución del problema }\end{array}$ \\
\hline 10 & $\begin{array}{l}\text { Responsabilidad social y } \\
\text { ambiental - RSA }\end{array}$ & $\begin{array}{l}\text { Es la capacidad de vincular y/o concientizar en las actividades y prácticas } \\
\text { académicas a los estudiantes, pares o sociedad. }\end{array}$ \\
\hline 11 & Trabajo en equipo - TE: & $\begin{array}{l}\text { Es la capacidad de colaborar y cooperar con los demás de forma efectiva para } \\
\text { alcanzar los objetivos de la universidad. }\end{array}$ \\
\hline 12 & Liderazgo - LID & $\begin{array}{l}\text { Es la capacidad de dirigir a un grupo o equipo de trabajo, es decir, el deseo de } \\
\text { guiar a los demás; de crear un compromiso, transmitiendo la visión de la } \\
\text { universidad, proporcionando dirección y delegando responsabilidades, así } \\
\text { como procurar que las personas tengan una buena integración en el equipo de } \\
\text { trabajo }\end{array}$ \\
\hline 13 & $\begin{array}{l}\text { Orientación a resultados - } \\
\text { OR }\end{array}$ & $\begin{array}{l}\text { Es la capacidad de orientar todas las acciones al logro de uno o varios } \\
\text { objetivos planteados, actuando con rapidez y diligencia ante decisiones } \\
\text { importantes, con el fin de satisfacer las necesidades institucionales. }\end{array}$ \\
\hline 14 & Orientación al servicio - OS & $\begin{array}{l}\text { Implica un deseo de ayudar o servir a los demás, de satisfacer sus } \\
\text { necesidades. }\end{array}$ \\
\hline \multicolumn{3}{|c|}{ Pedagógicas } \\
\hline 15 & $\begin{array}{l}\text { Planificación y ejecución } \\
\text { micro curricular - PMIC }\end{array}$ & $\begin{array}{l}\text { Comprende la elaboración e implementación de actividades dentro de la } \\
\text { clase, bajo una estructura clara, lógica y organizada. }\end{array}$ \\
\hline 16 & $\begin{array}{l}\text { Atención a la diversidad - } \\
\text { AD }\end{array}$ & $\begin{array}{l}\text { Prevenir y dar respuesta a las necesidades, temporales o permanentes, de los } \\
\text { estudiantes de la institución y, entre ellos, a los que requieren una actuación } \\
\text { específica derivada de diversos factores. }\end{array}$ \\
\hline 17 & $\begin{array}{l}\text { Manejo de herramientas y } \\
\text { técnicas de evaluación - } \\
\text { MHTE }\end{array}$ & $\begin{array}{l}\text { Es la capacidad de buscar parámetros de evaluación sobre la generación de } \\
\text { conocimiento de los alumnos en el aula, encontrando las mejores prácticas y } \\
\text { la adecuada ejecución de las herramientas pedagógicas }\end{array}$ \\
\hline 18 & $\begin{array}{l}\text { Estrategia metodológica } \\
\text { (acordes al área del saber) - } \\
\text { EM }\end{array}$ & $\begin{array}{l}\text { Es la capacidad de utilizar las estrategias que tiene la pedagogía en el } \\
\text { desarrollo de la docencia, con el propósito de crear conciencia participativa } \\
\text { en los estudiantes y el desarrollo de nuevas propuestas a situaciones propias } \\
\text { del pensamiento }\end{array}$ \\
\hline 19 & $\begin{array}{l}\text { Generación de un ambiente } \\
\text { participativo (intercambio } \\
\text { de experiencias) - GAP }\end{array}$ & $\begin{array}{l}\text { Es la capacidad de proponer diferentes maneras de trabajar en el aula desde } \\
\text { un enfoque participativo y grupal, considerando experiencias y/o vivencias } \\
\text { como herramienta para generar reflexiones y conocimiento }\end{array}$ \\
\hline
\end{tabular}
Fuente: (PUCE, 2018)

El perfil de competencias descrito, involucra de manera directa aspectos relacionados al desempeño general del docente y a cuestiones de su práctica pedagógica en las aulas de clases, pero deja un poco de lado aquellas fortalezas o virtudes personales que los seres humanos poseen y que los definen como personas, situación importante que se no solo complementaría el perfil 
del docente de la PUCE, sino que propendería a que, como persona, el docente sea íntegro, pleno y feliz.

\section{Fortalezas de carácter}

En un estudio realizado por Seligman y Peterson (2004), se considera que las fortalezas de carácter son rasgos y características psicológicas que se evidencian en distintos momentos y circunstancias a lo largo de la vida de una persona, son adquiribles y muestran el camino para alcanzar las seis virtudes humanas determinadas a través de dicho estudio. Se tomaron para esa investigación, varios personajes de la historia que mostraron aspectos coincidentes; de allí nacieron las 6 virtudes y 24 subcategorías de fortalezas humanas positivas. A éstas se les dio el carácter universal (Calderón, Forés Y Gustems, 2016), pues existen evidencias que indican que son rasgos característicos de personas de aproximadamente 54 países en los 5 continentes (Biswas-Diener, 2006; Park, Peterson y Seligman, 2006).

En el estudio de Seligman y Peterson (2004), se determinó que existen aquellas fortalezas de firma que son de tres a siete fortalezas que todas las personas las tienen más desarrolladas, son parte de su identidad y su autenticidad y "son el resultado de la búsqueda de los aspectos más valiosos y deseables del ser humano mediante el empleo de diferentes procedimientos empíricos" (Perandones, Lledó Y Grau, 210, p. 18).

A continuación, se detallan en la tabla 2, las virtudes, fortalezas de carácter y un descriptor que permite comprender cómo se evidencian éstas en las personas.

Tabla. 2

Virtudes y Fortalezas de carácter

\begin{tabular}{|c|c|c|c|}
\hline Virtud & & Fortaleza & Definición \\
\hline \multirow[t]{5}{*}{$\begin{array}{l}\text { Sabiduría } \\
\text { Conocimiento }\end{array}$} & $\mathbf{y}$ & $\begin{array}{l}\text { Creatividad - } \\
\text { Originalidad - } \\
\text { Ingenio }\end{array}$ & $\begin{array}{l}\text { Apertura a nuevas experiencias, flexibilidad cognitiva. Un } \\
\text { comportamiento diferente pero adecuado a su objetivo. }\end{array}$ \\
\hline & & Curiosidad & Explorar y descubrir. Compromiso y logro en el entorno académico \\
\hline & & $\begin{array}{l}\text { Criterio - Mente } \\
\text { abierta }\end{array}$ & $\begin{array}{l}\text { Pensar sobre las cosas y examinar todos sus significados y matices. } \\
\text { No sacar conclusiones al azar, sino tras evaluar cada posibilidad. } \\
\text { Estar dispuesto a cambiar las propias ideas en base a la evidencia } \\
\text { Discernimiento, pensamiento crítico, lucidez. Hábil al solucionar } \\
\text { problemas. }\end{array}$ \\
\hline & & Amor al aprendizaje & $\begin{array}{l}\text { Hábil al atravesar obstáculos y retos, autonomía, ingenio, } \\
\text { autoeficacia, envejecimiento sano y productivo. }\end{array}$ \\
\hline & & $\begin{array}{l}\text { Perspectiva - } \\
\text { Sabiduría }\end{array}$ & $\begin{array}{l}\text { Buen nivel de autoconocimiento; decisiones basadas tanto en la } \\
\text { razón como en la emoción, siempre pautadas en las necesidades de } \\
\text { los otros; madurez, sociabilidad. }\end{array}$ \\
\hline \multirow[t]{4}{*}{ Coraje } & & Valentía & $\begin{array}{l}\text { Orientación pro social, capacidad para evaluar riesgos, capacidad } \\
\text { para crear y sostener vínculos de calidad con otros. }\end{array}$ \\
\hline & & Perseverancia & $\begin{array}{l}\text { Terminar lo que se propuso hacer. Hacer con placer y con buen } \\
\text { humor; es flexible y realista; consecución de logros y objetivos, } \\
\text { Ingenio. }\end{array}$ \\
\hline & & $\begin{array}{l}\text { Integridad - } \\
\text { autenticidad }\end{array}$ & $\begin{array}{l}\text { Su código de valores guía y da sentido a su vida; capacidad de auto } \\
\text { actualización, diligencia, estabilidad emocional. }\end{array}$ \\
\hline & & $\begin{array}{l}\text { Vitalidad - } \\
\text { entusiasmo - energía }\end{array}$ & $\begin{array}{l}\text { La persona se siente viva, animada, energizada; casi siempre se } \\
\text { siente despierta y activa, vínculo con los demás, autonomía. }\end{array}$ \\
\hline
\end{tabular}




\begin{tabular}{|c|c|c|}
\hline Virtud & Fortaleza & Definición \\
\hline \multirow[t]{3}{*}{ Humanidad } & $\begin{array}{l}\text { Amor - capacidad de } \\
\text { amar y ser amado }\end{array}$ & $\begin{array}{l}\text { Relaciones positivas con los demás, balance sano entre dependencia } \\
\text { y autonomía, funcionamiento social positivo, autoestima alta. }\end{array}$ \\
\hline & $\begin{array}{l}\text { Generosidad - } \\
\text { gentileza - bondad }\end{array}$ & $\begin{array}{l}\text { Dar es más importante que recibir. Ser bueno y misericordioso con } \\
\text { las personas, estar preocupado por su bienestar. }\end{array}$ \\
\hline & $\begin{array}{l}\text { Inteligencia social - } \\
\text { inteligencia } \\
\text { emocional }\end{array}$ & $\begin{array}{l}\text { Habilidad de procesar información emocional, capacidad de } \\
\text { entender y administrar emociones, funcionamiento social fluido. }\end{array}$ \\
\hline \multirow[t]{3}{*}{ Justicia } & $\begin{array}{l}\text { Ciudadanía - Trabajo } \\
\text { en equipo }\end{array}$ & $\begin{array}{l}\text { Responsabilidad por mejorar el mundo en el cual vive, confianza } \\
\text { social, opinión positiva de la vida. }\end{array}$ \\
\hline & $\begin{array}{l}\text { Justicia - equidad - } \\
\text { imparcialidad }\end{array}$ & $\begin{array}{l}\text { Tratar a las personas de modo similar o idéntico, ser comprometido } \\
\text { con la idea de que las mismas reglas se aplican a todos. }\end{array}$ \\
\hline & Liderazgo & $\begin{array}{l}\text { Capacidad de planear una línea de acción para motivar a otros para } \\
\text { desempeñar mejor una tarea; estabilidad emocional, habilidades } \\
\text { interpersonales. }\end{array}$ \\
\hline \multirow[t]{4}{*}{ Templanza } & Perdón y misericordia & $\begin{array}{l}\text { Afabilidad, estabilidad emocional, logran rehacerse rápidamente } \\
\text { ante la decepción. }\end{array}$ \\
\hline & Humildad y modestia & $\begin{array}{l}\text { Estilo de comportamiento discreto, conciencia de que la persona no } \\
\text { es el centro del universo. }\end{array}$ \\
\hline & Prudencia & $\begin{array}{l}\text { Disposición de cooperar, capacidad de reflexión, no sacrifica } \\
\text { objetivos a largo plazo por placeres a corto plazo }\end{array}$ \\
\hline & $\begin{array}{l}\text { Autocontrol - } \\
\text { disciplina - auto } \\
\text { regulación }\end{array}$ & $\begin{array}{l}\text { La persona ejerce control sobre sus respuestas, vive a la altura de } \\
\text { estándares, control de impulsos para un comportamiento moral. }\end{array}$ \\
\hline \multirow[t]{5}{*}{ Trascendencia } & $\begin{array}{l}\text { Apreciación de la } \\
\text { belleza y de la } \\
\text { excelencia }\end{array}$ & $\begin{array}{l}\text { Experiencia emocional de la reverencia, asombro y admiración en la } \\
\text { presencia de la belleza o la excelencia }\end{array}$ \\
\hline & Gratitud & Satisfacción con la vida, afabilidad, diligencia, conducta pro social. \\
\hline & Esperanza & $\begin{array}{l}\text { Optimismo, tendencia y orientación a la solución de problemas a } \\
\text { futuro. }\end{array}$ \\
\hline & Humor & $\begin{array}{l}\text { Ánimo positivo, capacidad de manejar el estrés, creatividad, } \\
\text { inteligencia. }\end{array}$ \\
\hline & $\begin{array}{l}\text { Espiritualidad - } \\
\text { Sentido de propósito }\end{array}$ & $\begin{array}{l}\text { Posesión de creencias coherentes con el significado del universo y } \\
\text { su lugar en él. Se conectan al interés en valores morales y la } \\
\text { búsqueda del bien. }\end{array}$ \\
\hline
\end{tabular}

Fuente: (Seligman \& Peterson, 2004), (De Sá, 2019)

Según el estudio antes citado, las fortalezas de carácter se manifiestan en distintas situaciones, a través de pensamientos, sentimientos y acciones, pero algo interesante, es que son maleables a través del tiempo y de la vida de una persona, sujeto a influencias de factores externos en los que se desarrolla, además de que se pueden medir.

Si bien, todas las fortalezas están presentes en mayor o menor medida en una persona, en el contexto del docente de la PUCESA, que está involucrado en un marco académico y humanista en búsqueda de la excelencia de sus estudiantes, se hace necesario identificar las fortalezas de carácter que deben trabajarse en conjunto con los valores y que idealmente se requerirían en el perfil del maestro, de tal manera que partiendo de una línea base, se ejecuten actividades y se propongan planes de desarrollo docente que permitan que dichas fortalezas se manifiesten en beneficio personal, de los estudiantes y de la institución. 


\section{Metodología}

La investigación tiene un alcance exploratorio, por cuanto no existe información que relacione la psicología positiva y las fortalezas de carácter con el perfil de un docente universitario, y también un alcance descriptivo, puesto que ha sido posible a través de este estudio, mostrar algunas dimensiones interesantes respecto al contexto de la investigación, desde el punto de vista legal en lo que corresponde a las normativas involucradas y desde el punto de vista técnico, referente al modelo y a los procesos de evaluación del personal académico.

Además, tiene un enfoque mixto basado en una investigación no experimental, en donde se utilizó el análisis de información en lo que respecta a los reglamentos, perfiles docentes y modelos, y se aplicó un instrumento de cotejo a una muestra de docentes de la PUCESA para recopilar información como insumos para la propuesta.

En la primera parte del estudio se realizó el análisis de la información referente al modelo de evaluación docente de la PUCE, enfocado en la evaluación por competencias, en donde fue necesario además analizar el perfil de competencias del docente de la institución, detectándose que existe una falencia en la valoración de la parte humana del docente, por otra parte, se revisó a profundidad la propuesta de Selligman (2004), respecto a las fortalezas de carácter, y con la aplicación de una encuesta a una muestra de docentes de la PUCESA, se pudo definir la propuesta en la primera parte, que corresponde a complementar el perfil del docente involucrando las fortalezas de carácter necesarias.

En una segunda parte, se plantea una propuesta referente a establecer las fortalezas de carácter que deberían tener desarrolladas tanto los evaluadores como los evaluados para alcanzar mejores resultados y se proponen un conjunto de actividades tendientes a desarrollar o potenciar esas fortalezas, tomando como base los postulados de la psicología positiva.

\section{Resultados y discusión}

En una primera parte de la propuesta, para establecer las fortalezas de carácter que están relacionadas con el perfil del docente de la PUCESA, se tomó como base el estudio publicado por Perandones, Herrera \& Lledó (2014), acerca de las fortalezas y virtudes personales del profesorado y su relación con la eficacia docente, en donde se resalta la capacidad que tiene el docente para ejercer una influencia positiva sobre el aprendizaje de sus estudiantes, influencia que va orientada a ayudarles a los estudiantes a conseguir sus objetivos, pero también tendrán una relación de influencia, los pensamientos, acciones y emociones que los docentes generen en ellos; es el momento en el que las creencias y actitudes de los docentes guardan una estrecha relación con sus prácticas de enseñanza

Dicho estudio demuestra que "los profesores con elevado sentimiento de autoeficacia manifiestan mucho entusiasmo por enseñar y se encuentran más comprometidos con su profesión, de modo que muestran mayor apertura a nuevas ideas, están dispuestos a probar métodos innovadores, planifican y organizan mejor sus clases y dedican más tiempo a los estudiantes" (Perandones et. al., 2014, p. 145). 
En vista de lo anteriormente expuesto, se hace indispensable resaltar las características personales de los docentes, dentro de su perfil requerido. Para ello, se ha tomado como base el perfil de competencias del Docente de la PUCE y las virtudes y fortalezas de carácter propuestas por Seligman \& Peterson (2004), con sus respectivos descriptores, para contrastar la información y seleccionar las fortalezas que deben caracterizar a un Docente de la PUCE.

Se aplicó un instrumento de cotejo a una muestra de 31 docentes de la PUCESA de un total de 104 docentes titulares, en donde tomando como base la información descrita en el párrafo anterior, se les pidió analizar y seleccionar aquellas fortalezas de carácter que consideran se relacionan con las competencias dentro del perfil del docente dando como resultado lo siguiente:

Tabla. 3

Perfil de competencias y fortalezas del docente de la PUCE

Competencias Docente PUCE

Fortalezas De Carácter Relacionadas Con Las Competencias Del Docente De La PUCESA

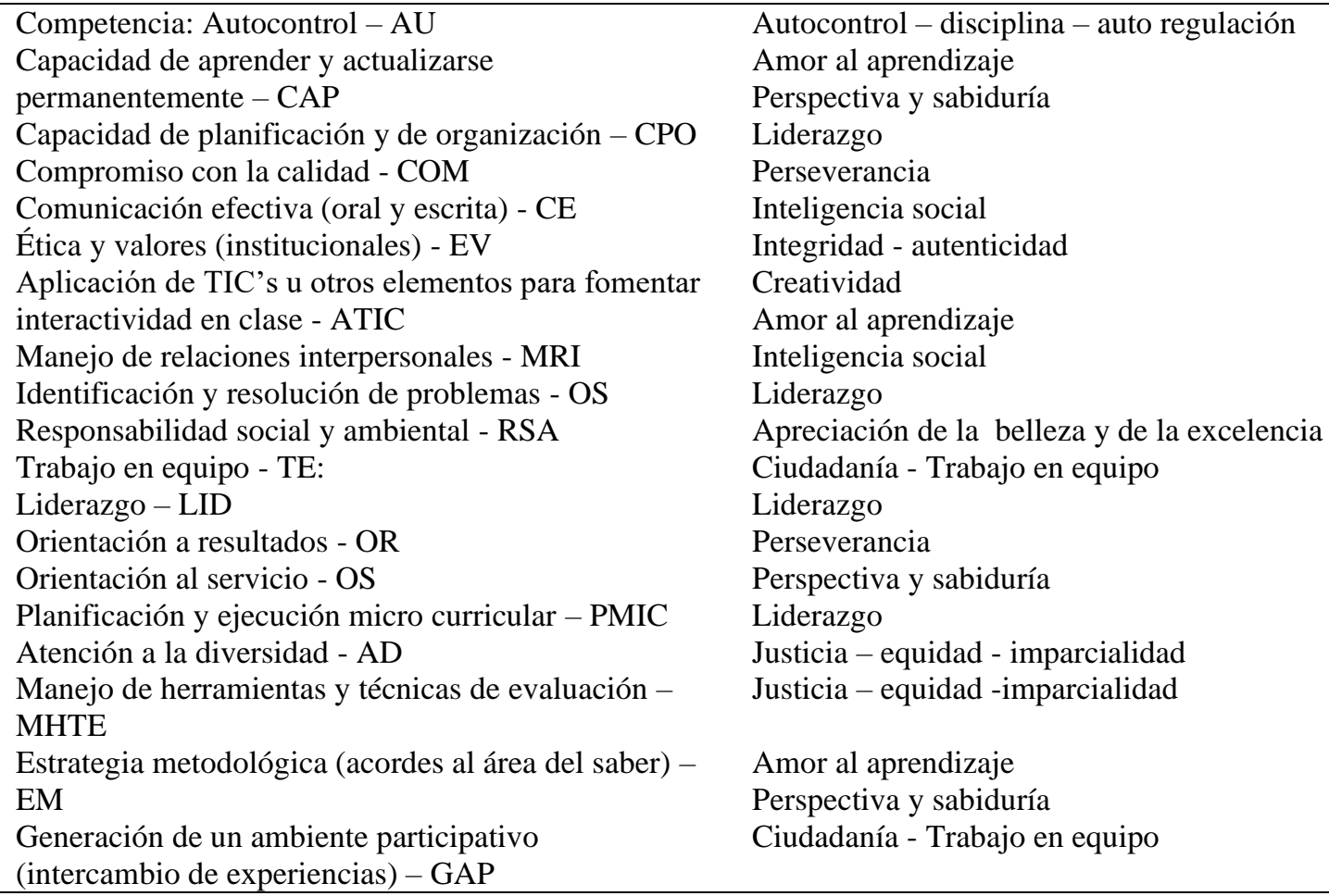

Fuente: elaboración propia

A continuación, y tomando como referencia el estudio de Seligman y Peterson (2004), se definen las fortalezas de firma que deberían caracterizar al docente de la PUCESA, para ello, se presenta una tabla que muestra la frecuencia de aparición de las fortalezas de carácter en relación con las competencias, con un resumen de las virtudes con las fortalezas de carácter que, según los resultados obtenidos, tienen relación con el perfil actual del docente de la PUCE, cabe indicar que no se colocan aquellas fortalezas que no se reflejan en el perfil de competencias, pues lo que se pretende resaltar son aquellas que más desarrolladas debería tener el docente y deberían caracterizarlo: 
Tabla. 4

Fortalezas relacionadas al perfil del docente de la PUCE

\begin{tabular}{|c|c|c|c|}
\hline Virtud & & Fortaleza & $\begin{array}{c}\text { Presencia De La Fortaleza En EI Perfil Del Docente } \\
\text { De La Puce (Frecuencia) }\end{array}$ \\
\hline Sabiduría & $\mathrm{Y}$ & Creatividad y Originalidad & 1 \\
\hline \multirow[t]{2}{*}{ Conocimiento } & & Amor al aprendizaje & 3 \\
\hline & & Perspectiva y sabiduría & 3 \\
\hline \multirow[t]{2}{*}{ Coraje } & & Perseverancia & 2 \\
\hline & & Integridad - autenticidad & 1 \\
\hline Humanidad & & Inteligencia social & 2 \\
\hline \multirow[t]{3}{*}{ Justicia } & & Ciudadanía - Trabajo en equipo & 2 \\
\hline & & $\begin{array}{l}\text { Justicia }- \\
\text { imparcialidad }\end{array}$ & 2 \\
\hline & & Liderazgo & 4 \\
\hline Templanza & & $\begin{array}{l}\text { Autocontrol - disciplina - auto } \\
\text { regulación }\end{array}$ & 1 \\
\hline Trascendencia & & $\begin{array}{l}\text { Apreciación de la belleza y de } \\
\text { la excelencia }\end{array}$ & 1 \\
\hline
\end{tabular}

Fuente: elaboración propia

Los resultados muestran que 11 de las 24 fortalezas de carácter, idealmente un docente de la PUCE debería tenerlas más desarrolladas dentro de su rol académico, investigativo y de gestión, sin dejar de lado las que no aparecen en la tabla ya que como se explicó anteriormente, éstas se modifican y priorizan según los cambios y necesidades del contexto en el que se desarrolla una persona, y en este caso estaría relacionado directamente a la institución.

En concordancia con el estudio de Perandones et. al. (2014), finalmente los resultados mostrados evidencian que para desempeñar con éxito sus funciones, el docente no solo requiere de competencias profesionales sino también personales, las que están enmarcadas en "comportamientos pro sociales, autoestima, liderazgo, ..., relacionado a mayores niveles de realización personal y profesional” (Seligman, Ernst, Gillham, Reivich, \& Linkins, 2009).

El esquema ideal entonces, sería que el perfil del docente de la PUCE incorpore a sus competencias, las fortalezas de carácter propuestas en el análisis, luego de la respectiva socialización y validación en las instancias que sean necesarias para realizar los ajustes pertinentes, situación que tendría además, una enorme implicación en decisiones futuras que puedan tomarse, tales como en la formación docente, en donde el dar importancia a las virtudes y fortalezas de carácter tanto en la práctica docente como en las políticas educativas, llevaría a generar mayor bienestar y felicidad en los docentes (Gustems \& Sánchez, 2015).

En la segunda parte de la propuesta, a partir del análisis del MEIPA, además de la importancia que tiene involucrar las fortalezas de carácter en el perfil del docente, para resaltar la parte humana, se identificaron algunos aspectos importantes que, en base a la experiencia de los procesos ya aplicados, se considera contribuiría a incrementar el éxito y objetividad del proceso de evaluación docente en sus distintos momentos:

Autoevaluación: la capacidad de reflexión e interiorización que el docente tenga y que le permita de una forma crítica y objetiva revisar su actuar y su desempeño en las actividades a él asignadas. 
Co evaluación: relacionada directamente a los pares evaluadores en el caso de la evaluación en el ámbito de docencia, y de los directivos e investigadores para gestión académica e investigación y vinculación respectivamente; en donde, se hace necesaria una gran dosis de prudencia, responsabilidad y la integridad necesaria para observar de manera imparcial y humana el trabajo de los docentes a quienes van a evaluar.

Hetero evaluación: siendo éste el aspecto que mayor peso tiene en el proceso de evaluación y que les corresponde directamente a los estudiantes, es necesario que se desarrolle dentro de un marco de justicia y humanidad, pero sobre todo de honestidad para lograr que el momento de la evaluación se logren resultados lo más cercanos a la realidad.

Retroalimentación: que es sumamente importante y clave dentro del proceso de evaluación docente, pues es la instancia en la cual se pretende conseguir una reacción positiva y de mejora en los docentes respecto a los resultados alcanzados en su evaluación. Es allí en donde, el Humanismo Cristiano como la base del enfoque filosófico general de la PUCE, debe entrar en acción reconociendo al docente como "un ser humano que posee una especial dignidad y valor que lo distingue de los demás” (PUCE, 2017), y que quienes están inmersos en la retroalimentación deben propender a reducir tensiones que pongan en riesgo la integridad y dignidad del docente, así como la objetividad y propósito de la evaluación.

Frente al análisis realizado, se puede deducir que, se requiere que quienes estén involucrados en el proceso en calidad de evaluadores y evaluados tengan desarrolladas ciertas características que, de manera personal y no tanto técnica, les permita desarrollar un buen papel y obtener resultados objetivos y válidos para tomar decisiones en la institución educativa.

Al respecto, con el enfoque de la propuesta, se pretende además fortalecer el proceso de evaluación docente mediante el planteamiento de actividades orientadas a desarrollar determinadas fortalezas de carácter en los evaluados y evaluadores dentro de los momentos de autoevaluación, co evaluación, hetero evaluación y retroalimentación, en función de aquello, los destinatarios de esta parte de la propuesta son todos los actores del proceso de evaluación docente, resumidos en la siguiente tabla: 
Tabla. 5

Destinatarios de las actividades en los distintos momentos de evaluación

\begin{tabular}{|c|c|c|}
\hline $\begin{array}{l}\text { Momento Del } \\
\text { Proceso }\end{array}$ & Beneficiarios De La Propuesta & Ámbito \\
\hline Autoevaluación & $\begin{array}{c}\text { Docentes Tiempo completo, medio tiempo y } \\
\text { Tiempo parcial de las distintas Unidades } \\
\text { Académicas }\end{array}$ & $\begin{array}{c}\text { Fortalezas de carácter asociadas a } \\
\text { desempeñarse como evaluado y } \\
\text { evaluador }\end{array}$ \\
\hline \multirow[t]{4}{*}{ Co Evaluación } & $\begin{array}{c}\text { Pares Evaluadores de las distintas Unidades } \\
\text { Académicas }\end{array}$ & $\begin{array}{l}\text { Fortalezas de carácter asociadas a } \\
\text { desempeñarse como evaluador }\end{array}$ \\
\hline & $\begin{array}{l}\text { Directivos (Directores de Escuela de las } \\
\text { distintas Unidades Académicas) }\end{array}$ & $\begin{array}{l}\text { Fortalezas de carácter asociadas a } \\
\text { desempeñarse como evaluador }\end{array}$ \\
\hline & $\begin{array}{c}\text { Coordinadores de Proyectos de investigación y } \\
\text { vinculación }\end{array}$ & $\begin{array}{l}\text { Fortalezas de carácter asociadas a } \\
\text { desempeñarse como evaluador }\end{array}$ \\
\hline & $\begin{array}{c}\text { Docentes Tiempo completo, medio tiempo y } \\
\text { Tiempo parcial de las distintas Unidades } \\
\text { Académicas }\end{array}$ & $\begin{array}{l}\text { Fortalezas de carácter asociadas a } \\
\text { desempeñarse como evaluado }\end{array}$ \\
\hline Hetero Evaluación & $\begin{array}{c}\text { Estudiantes de Pregrado de las distintas } \\
\text { Unidades Académicas }\end{array}$ & $\begin{array}{l}\text { Fortalezas de carácter asociadas a } \\
\text { desempeñarse como evaluador }\end{array}$ \\
\hline & $\begin{array}{c}\text { Docentes Tiempo completo, medio tiempo y } \\
\text { Tiempo parcial de las distintas Unidades } \\
\text { Académicas }\end{array}$ & $\begin{array}{l}\text { Fortalezas de carácter asociadas a } \\
\text { desempeñarse como evaluado }\end{array}$ \\
\hline \multirow[t]{2}{*}{ Retroalimentación } & $\begin{array}{c}\text { Directivos (Director Académico y Directores } \\
\text { de Escuela) }\end{array}$ & $\begin{array}{l}\text { Fortalezas de carácter asociadas al } \\
\text { proceso de retroalimentación }\end{array}$ \\
\hline & $\begin{array}{l}\text { Docentes Tiempo completo, medio tiempo y } \\
\text { Tiempo parcial de las distintas Unidades } \\
\text { Académicas }\end{array}$ & $\begin{array}{l}\text { Fortalezas de carácter asociadas al } \\
\text { proceso de retroalimentación }\end{array}$ \\
\hline
\end{tabular}

Fuente: elaboración propia

A continuación, se presentan las fortalezas de carácter identificadas, para los roles de evaluado y evaluador, que son desempeñados por los docentes, estudiantes, directivos, coordinadores de proyectos y pares evaluadores, en los distintos momentos de evaluación identificados como claves dentro del proceso: 
Tabla. 6

Fortalezas de Evaluados y Evaluadores en los momentos de la evaluación

\begin{tabular}{|c|c|c|c|c|}
\hline Momento & Evaluador & $\begin{array}{c}\text { Fortaleza } \\
\text { A Trabajar }\end{array}$ & Evaluado & Fortaleza A Trabajar \\
\hline Autoevaluación & Docente & $\begin{array}{l}\text {-Justicia - equidad - } \\
\text { Imparcialidad }\end{array}$ & Docente & -Criterio / Mente abierta \\
\hline $\begin{array}{c}\text { Hetero } \\
\text { evaluación }\end{array}$ & Estudiantes & $\begin{array}{l}\text {-Justicia - equidad - } \\
\text { Imparcialidad }\end{array}$ & Docente & $\begin{array}{l}\text {-Autocontrol - } \\
\text { disciplina/Auto regulación }\end{array}$ \\
\hline \multirow[t]{5}{*}{ Co-evaluación } & $\begin{array}{l}\text { Docentes (Par } \\
\text { evaluador) }\end{array}$ & $\begin{array}{l}\text {-Autocontrol - } \\
\text { disciplina/Auto regulación }\end{array}$ & Docente & $\begin{array}{l}\text {-Autocontrol - } \\
\text { disciplina/Auto regulación }\end{array}$ \\
\hline & & $\begin{array}{l}\text {-Justicia - equidad - } \\
\text { Imparcialidad }\end{array}$ & & \\
\hline & Directivos & -Inteligencia Social & Docente & $\begin{array}{l}\text {-Autocontrol - } \\
\text { disciplina/Auto regulación }\end{array}$ \\
\hline & & $\begin{array}{l}\text {-Justicia - equidad - } \\
\text { Imparcialidad }\end{array}$ & & \\
\hline & $\begin{array}{l}\text { Coordinador Proyecto } \\
\text { investigación }\end{array}$ & -Liderazgo & Docente & $\begin{array}{l}\text {-Autocontrol - } \\
\text { disciplina/Auto regulación }\end{array}$ \\
\hline
\end{tabular}

Fuente: elaboración propia

La retroalimentación se la ha separado de la tabla anterior por ser un proceso distinto que concluye la evaluación integral del personal académico, pero dentro del nivel de importancia, posiblemente la que más peso tendría en la parte final.

Tabla.7

Fortalezas a trabajar en la retroalimentación

\begin{tabular}{ccccc}
\hline Momento & Entrega & Fortaleza & Recibe & Fortaleza \\
\hline Retroalimentación & Director Académico/ & -Inteligencia social & Docente & - Autocontrol - \\
& Director de Escuela & & & disciplina/Auto regulación \\
& & -Justicia - equidad - & & \\
& & Imparcialidad & \\
\hline
\end{tabular}

Fuente: elaboración propia

La propuesta refleja además un conjunto de actividades a desarrollar en los momentos del proceso de evaluación docente que se han mencionado, adicionalmente serán dirigidas particularmente a cada uno de los actores de dicho proceso, con el fin de potenciar las fortalezas identificadas y necesarias en cada momento.

Son un total de 6 actividades que se han detallado en un formato diseñado para el efecto y que contiene todos los elementos que se cree son necesarios para que se puedan aplicar de una manera sencilla y práctica.

A continuación, se muestra un ejemplo de la actividad 1, dirigida a los Docentes para el proceso de Auto evaluación docente: 


\begin{tabular}{|c|c|c|c|c|c|}
\hline \multicolumn{6}{|c|}{ ACTIVIDAD 1} \\
\hline \multicolumn{6}{|c|}{ FORTALEZA A POTENCIAR: Criterio / Mente abierta } \\
\hline \multicolumn{3}{|c|}{ MOMENTO: Autoevaluación Docente } & \multicolumn{3}{|c|}{ Dirigido a: Docentes } \\
\hline \multicolumn{6}{|c|}{$\begin{array}{l}\text { Descripción de la Fortaleza: } \\
\text { Pensar sobre las cosas y examinar todos sus significados y matices. No sacar conclusiones al azar, sino tras evaluar cada posibilidad. Estar dispuesto a cambiar las propias } \\
\text { ideas en base a la evidencia. Discernimiento, pensamiento crítico, lucidez. }\end{array}$} \\
\hline \multicolumn{3}{|c|}{ Período de Ejecución: 1 mes (un mes antes de iniciar el proceso de evaluación docente } & \multicolumn{3}{|c|}{ Frecuencia de Ejecución: Semanal (1 vez por semana) -total 4} \\
\hline \multicolumn{6}{|c|}{ Evidencia de cumplimiento: Documentos de anotaciones de los ejercicios realizados por los docentes } \\
\hline TEMA & OBJETIVOS & DESCRIPCIÓN & TIEMPO & CONCLUSIONES & MATERIALES \\
\hline Reflexión Docente & $\begin{array}{l}\text { 1. Analizar nuestra práctica } \\
\text { docente en las aulas de } \\
\text { clases para identificar } \\
\text { aquellos aspectos } \\
\text { positivos y negativos que } \\
\text { se reflejan en nuestro } \\
\text { actuar, reflexionando } \\
\text { sobre lo que causa que } \\
\text { aparezcan más a menudo } \\
\text { y con mayor intensidad. } \\
\text { 2. Tener conciencia plena } \\
\text { de mi desempeño en las } \\
\text { aulas de clases para una } \\
\text { mejora continua. }\end{array}$ & $\begin{array}{l}\text { Durante un mes ( } 4 \text { semanas), al } \\
\text { finalizar cada semana, } \\
\text { anotaremos en un cuadro la } \\
\text { siguiente información: } \\
\text { 1. Día y hora } \\
\text { Fortalezas } \\
\text { 2. ¿Qué cosas positivas puedo } \\
\text { identificar en mis clases de } \\
\text { esta semana? } \\
\text { 3. ¿Qué causó que fuera así? } \\
\text { 4. ¿Cómo me siento al } \\
\text { respecto? } \\
\text { Debilidades } \\
\text { 5. ¿En qué aspectos no me } \\
\text { desempeñé de lo mejor esta } \\
\text { semana? } \\
\text { 6. ¿Por qué no lo hice? } \\
\text { 7. ¿Con cuánta frecuencia me } \\
\text { ocurre ésto? } \\
\text { 8. ¿Cómo me siento al } \\
\text { respecto? } \\
\text { 9. ¿Está en mis manos } \\
\text { cambiar esas situaciones? }\end{array}$ & 15 minutos & $\begin{array}{l}\text { Habitualmente } \\
\text { nuestras actividades } \\
\text { en las aulas de clases, } \\
\text { se vuelven } \\
\text { automáticas y } \\
\text { rutinarias y las vemos } \\
\text { como razonables y } \\
\text { correctas porque no } \\
\text { reflexionamos sobre } \\
\text { su desarrollo y sus } \\
\text { resultados, sin tomar } \\
\text { conciencia. } \\
\text { Esta actividad nos } \\
\text { permite visualizar } \\
\text { especialmente los } \\
\text { aspectos negativos } \\
\text { analizando sus causas } \\
\text { para poder actuar } \\
\text { inmediatamente. }\end{array}$ & $\begin{array}{ll}\checkmark & \text { Papel } \\
\checkmark & \text { Esfero }\end{array}$ \\
\hline
\end{tabular}

Figura \#2 muestra el detalle de la actividad \#1 diseñada con los elementos necesarios para que el docente pueda desarrollarla en su rol de evaluador dentro del momento de auto evaluación.

En la figura se aprecian elementos importantes como la fortaleza que se desea potencializar, el período y frecuencia de ejecución que son importantes para lograr que la actividad cumpla su objetivo, la evidencia de cumplimiento que serviría a futuro para verificar el desarrollo de las actividades como base de la medición de impacto, los objetivos y la descripción que son los que guían el desarrollo de la actividad en sí, el tiempo de duración que ha sido planteado de una manera razonable para evitar incomodar a quien lo aplica en sus actividades cotidianas, y finalmente las conclusiones y los materiales requeridos.

Como la actividad antes descrita se han propuesto 5 actividades más, en concordancia a lo establecido en la Tabla 6.

Para la aplicación de esta segunda parte de la propuesta sería importante incorporar las actividades al MEIPA, en la parte pertinente con el fin de aportar con los principios de la psicología positiva al proceso de evaluación docente.

\section{Conclusiones}

La evaluación docente en una institución de educación superior, al ser un proceso integral, debe incorporar aspectos que garanticen la valoración técnica, en cuanto a las competencias que el docente debe desarrollar dentro del ámbito profesional, así como procurar que la parte humana y social estén cubiertas para lograr que el resultado sea objetivo, y los procesos desarrollados le den al docente espacios propicios para tener un crecimiento continuo.

Es importante que dentro del perfil de un docente se contemplen las fortalezas de carácter necesarias para que las competencias profesionales se desarrollen dentro de una marco positivo y humano, que le permitan al docente realizarse de manera integral. 
Las actividades diseñadas para desarrollar las fortalezas de carácter en los evaluadores y evaluados en los distintos momentos de evaluación están fundamentadas en los principios de la psicología positiva que buscan el bienestar psicológico de quienes las desarrollan, con el fin de mejorar la percepción de dicho proceso.

La institución educativa, deben incorporar dentro de sus programas de desarrollo docente, actividades o capacitaciones orientadas al desarrollo de las fortalezas de carácter enunciadas en el perfil propuesto, para contribuir a la necesidad de tener docentes capacitados de manera integral que se sientan realizados, plenos y felices.

Es fundamental que los procesos de evaluación docente se desarrollen dentro de un marco adecuado y motivador para que los docentes alcancen estándares de una manera comprometida con la institución.

\section{Bibliografía}

Biswas-Diener, R. (2006). From the equator to the North Pole: A study of character strengths. Journal of Happiness Studies, 7, 293-310.

Blázquez, A. P. J., \& Casse, T. J. L. (2007). Modelo para autoevaluar la práctica docente. Recuperado de: https://ebookcentral-proquestcom.pucesa.idm.oclc.org/lib/pucesp/reader.action?docID=4870908\&ppg=16

Calderón, D., Forés, A. Y Gustems, J. (2016). Aproximación a las fortalezas de carácter en los estudiantes de Educación Social. Perfil de una muestra. Revista d'Innovació i Recerca en Educación. (9)2. ISSN-2013-2255. DOI: 10.1344/reire2016.9.2924

Cataluña, D. (2017). Manual de ejercicios de Psicología Positiva Aplicada. Colegio Oficial de Psicólogos de Madrid. ISBN: 978-84-87556-68-5. Recuperado de: https://www.copcv.org/db/docu/170419111407WtnYiyZunKZr.pdf

Consejo de Aseguramiento de la Calidad de la Educación Superior (2018), Modelo de Evaluación Institucional de Universidades y Escuelas Politécnicas 2018. Recuperado de: https://www.caces.gob.ec/

De Sá, R. (2019). Virtudes y Fortalezas de carácter. Primera Certificación Internacional en Educación Positiva Aplicada - Módulo 4.

Gustems, J., Sánchez, L. (2015). Aportaciones de la Psicología Positiva aplicadas a la formación del profesorado. Estudios sobre Educación, 29, 9-28. DOI: 10.15581/004.29.9-28.

Park, N., Peterson, C., y Seligman, M. E. P. (2006). Character strengths in fifty-four nations and the fifty US states. The Journal of Positive Psychology, 1(3), 118-129. DOI: 10.1080/17439760600619567.

Perandones, T., Lledó, A., Y Grau, S. (2010). Contribuciones de la psicología positiva al ámbito de la profesión docente. International Journal of Developmental and Educational Psychology. 1(1). ISSN 0214-9877

Pontificia Universidad Católica del Ecuador (2018). Diccionario de Competencias del Personal Académico de la PUCE.

Pontificia Universidad Católica del Ecuador (2018). Modelo de Evaluación Integral del Personal Académico (MEIPA).

Esta obra se comparte bajo la licencia Creative Common Atribución-No Comercial 4.0 International (CC BY-NC 4.0) 
Pontificia Universidad Católica del Ecuador (2017). Modelo Educativo de la Pontificia Universidad Católica del Ecuador.

Pontificia Universidad Católica del Ecuador (2017). Reglamento General de Personal Académico y Escalafón de la PUCE.

Proctor, C., Maltby, J., y Linley, P. A. (2011). Strengths use as a predictor of wellbeing and health-related quality of life. Journal of Happiness Studies, 12, 153-169.

Seligman, M. E. P., Ernst, R. M., Gillham, J., Reivich, K., \& Linkins, M. (2009). Positive education: Positive psychology and classroom interventions. Oxford Review of Education, 35 (3), 293-311. 